\title{
Prueba piloto de señalización en la Escuela Superior Politécnica del Chimborazo pintura fotoluminiscente
}

\author{
Signaling pilot test at the Chimborazo Higher Education School painting \\ fotoluminiscente
}

José Luis Llamuca Llamuca. ${ }^{1}$, Miriam del Rocío Salas Salazar. ${ }^{2}$ \& Alexandra Patricia Guerrero Godoy. ${ }^{3}$

\begin{abstract}
.
DOI: https://doi.org/10.33262/cienciadigital.v4i3.1319 Research on the relationship between horizontal signage and road safety within the premises of the Chimborazo Polytechnic Higher School located in the Lizarzaburu parish in the Riobamba canton.

For this reason, the analysis is focused on measuring the impact that horizontal signage has on road safety for people who make up the polytechnic community; as well as the drivers of the different motorized and non-motorized vehicles that interact in the daily actions of this higher education center; and generate the technical proposal for horizontal signaling on internal roads and thus contribute to the improvement of mobility and therefore the quality of life of those involved through the reduction of incidents in the area of road safety.
\end{abstract}

Keywords: Signaling, passenger transport, Transportation, roads, mobility.

\section{Resumen.}

La investigación sobre la relación que existe entre la señalización horizontal y la seguridad vial dentro de los predios de La Escuela superior Politécnica de Chimborazo ubicada en la parroquia Lizarzaburu del cantón Riobamba.

\footnotetext{
${ }^{1}$ Escuela Superior Politécnica del Chimborazo, Facultad de Administración de Empresas Escuela Gestión de Transporte, Riobamba Ecuador, jose.llamuca@espoch.edu.com

2 Escuela Superior Politécnica del Chimborazo, Facultad de Administración de Empresas Escuela Gestión de Transporte; Riobamba Ecuador, miriam.salas@espoch.edu.ec

${ }^{3}$ Escuela Superior Politécnica del Chimborazo, Facultad de Administración de Empresas Escuela Gestión de Transporte, Riobamba Ecuador, alexandra.guerrero@espoch.edu.com
} 
Por este motivo, el análisis está enfocado en medir el impacto que tiene la señalización horizontal en la seguridad vial de las personas que integran la comunidad politécnica; así como también a los conductores de los diferentes motorizados y no motorizados que interactúan en las acciones cotidianas de este centro de educación superior; y generar la propuesta técnica para la señalización horizontal en las vías internas y de esta manera contribuir a la mejora de la movilidad y por ende la calidad de vida de los involucrados a través de la disminución de incidentes en el área de seguridad vial, por esta razón queremos analizar la pintura fotoluminizente, con el fin de precautelar a la comunidad.

Palabras clave: Señalización, transporte de pasajeros, Transporte, vías, movilidad.

\section{Introducción.}

La señalización horizontal está acomodada, fundamentalmente, por las marcas viales. Éstas son unas marcas que se pintan directamente sobre la calzada, y su finalidad es la de advertir y guiar a los usuarios, así como regular el tráfico.

Son líneas o figuras, aplicadas sobre el pavimento, que tienen como objetivo satisfacer una o varias funciones.

Debido a su ubicación y sus características, las marcas viales son de vital importancia con condiciones meteorológicas adversas y de reducida visibilidad, por lo que su correcto mantenimiento y ejecución resultan cruciales para mantener la seguridad vial en dichas situaciones (por ejemplo, con niebla densa, el conductor puede tener fuera de su campo visual una señal vertical y, sin embargo, puede leerla si se ha pintado sobre la calzada, además de que las marcas longitudinales le ayudan a guiarse y mantenerse sobre la vía). Ingresan diariamente cerca de 20000 personas (según el último informe de talento Humano y registro de matrículas del periodo octubre 2017 - marzo 2018) entre estudiantes, docentes, empleados y trabajadores de la misma; la oferta académica de la institución se incrementa cada año, Nos enfocamos en medir el impacto que tiene la señalización horizontal en la seguridad vial de las personas que integran la comunidad politécnica.

\subsection{Planteamiento del problema}

La ESPOCH acuden diariamente cerca de 20000 personas (según el último informe de talento Humano y registro de matrículas del periodo Octubre 2017 - Marzo 2018) que se encuentran emparentadas con la actividad de educación superior ya sea como estudiantes, docentes, empleados y trabajadores de la misma; cada año esta cifra de personas sigue aumentando

Ocasionando que las instalaciones y los accesos viales a las mismas tengan que aumentarse considerablemente para brindar un servicio de educación acorde a la demanda presentada; pero ello ha provocado que algunos parámetros de movilidad como la señalización horizontal aun no brinden la garantía para contar con una seguridad vial 
adecuada dentro de las instalaciones de la ESPOCH. Or lo cual trabajamos el impacto que tiene la señalización horizontal en la seguridad vial de las personas que integran la comunidad politécnica.

\section{Justificación de la investigación.}

Recubrimiento diseñado en base de una resina acrílica base solvente de un solo componente. Su principal característica es su efecto fotoluminiscente, lo que genera luminosidad en la obscuridad después de estar expuesta a iluminación natural o artificial como lámparas y focos. (Pinturas Unidas, 2018)

Las opciones de transporte, especialmente la del transporte terrestre, están generando problemas negativos para el desarrollo de seguridad para los seres humanos, especialmente en el transporte situación que puede ser más compleja al no ejecutar planes de concientización.

El sistema de transporte terrestre de la ciudad de Riobamba, tiene ciertas características que convierten el servicio en una solución a medias, en mucho de los casos sin señalización eliminando la forma segura de transporte, no se observan los estándares de calidad, y sumada por diversas causas como por temas de conducta de los seres humanos en general (peatones y conductores) de aquí nace la importación de la señalética en forma general y por esta razón se quiere aplicar como prueba el uso de la pintura efecto fotoluminiscente para reducir los accidentes en la noche.

Por esta razón se pretende crear un plan piloto. En incremento en carretera urge una señalización diferente y segura pues coincide en la señalética en forma general tiene que estar muy bien resaltadas y en lugares visibles, esta investigación busca una nueva alternativa, para amenorar estos problemas en la señalización horizontal aplicando pues la composición química de esta pintura aplicada en este estudio, ayuda al conductor con una mejor visión sobre todo altas horas de la noche, la señalética utilizada en este ciudad no ayuda en la visión nocturna lo que provoca accidentes en aumento, y más cuando el parque automotor está en incremento esto reflejado en la ciudad.

\section{Marco teórico}

\section{Señalización}

Es el conjunto de estímulos que condiciona la actuación de las personas que los captan frente a determinadas situaciones que se pretender resaltar.

La señalización de seguridad tiene como misión llamar la atención sobre los objetos o situaciones que pueden provocar peligros, así como para indicar el emplazamiento de dispositivos y equipos que tengan importancia desde el punto de vista de seguridad en los centros locales de trabajo. 


\section{Transporte por carretera.}

En el transporte rápido especialmente concebido para disttúa dentro de la ciudad. $\mathrm{Su}$ principal razón de ser es proporcionar un servicio de apro (CerdA, 2017)visionamiento y distribución tanto a los establecimientos empresariales localizados en ella como al consumidor final. Pese a la escasez de información específica en el campo de la logística urbana, puede establecerse una relación directamente proporcional entre la actividad económica, el volumen de población y el volumen de la logística urbana generada, de manera que, conforme crecen la actividad económica y la población, aumenta la complejidad de la optimización del funcionamiento de la cadena de la logística urbana. Cabe destacar que la operativa asociada a esta actividad tiene un impacto sobre la movilidad de la ciudad y sobre el coste global de la cadena logística para los operadores.

Cerdà, I. (2010). Logística urbana. Barcelona, Spain: Marge Books. Recuperado de https://elibro.net/es/ereader/espoch/59295?page=11.ancias menores (Castro, 2017)

Clases de señalización

Del sentido al que se pretende sobresaltar, la señalización se clasifica en: señalización óptica, acústica, olfativa y táctil.

Tabla 1. Clases de señalización.

\begin{tabular}{l} 
Clases de señalización \\
\hline \\
Señales de seguridad. \\
Avisos de seguridad. \\
Óptica $\quad$ Colores de señalización. \\
\\
Balizamiento \\
Acústica \\
Olfativa \\
Táctil
\end{tabular}

Fuente: Elaboración propia.

\section{Definición de signo de seguridad.}

Sirviéndose de la combinación de una forma geométrica, un color y un emblema proporciona una investigación determinada relacionada con la seguridad.

\section{Clases de signos de seguridad.}

En función de su aplicación se dividen en: 
- Signos de prohibición: Señal de seguridad que impide un comportamiento que puede provocar una situación de peligro.

- Signos de obligación: Es un signo de seguridad que obliga a un comportamiento determinado.

- Signos de advertencia: Señal de seguridad que advierte un peligro.

- Signos de información: Señal que suministra información para facilitar el salvamento o garantizar la seguridad de las personas.

- Signo de salvamento: Es el signo que en caso de riesgo indica la salida de emergencia, la situación del puesto de socorro o el emplazamiento de un dispositivo de salvamento.

- Signo indicativo: Suministra otras investigaciones diferentes a las de prohibición, obligación y de advertencia.

- Signo auxiliar: Contienen únicamente texto y se maneja conjuntamente con las señales indicadas anteriormente.

- Señal complementaria de riesgo permanente: Sirven para señalizar lugares donde no se utilicen formas geométricas normalizadas y que suponen un riesgo permanente de choque, caída.

\section{Colores, formas, dimensiones y símbolos.}

Figura 1. Ángulos de iluminación

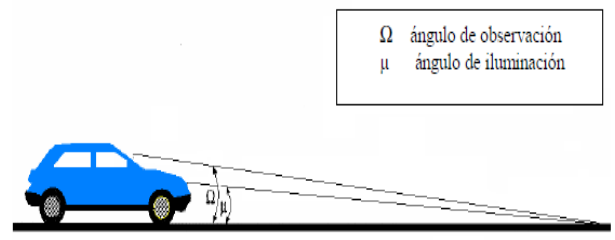

Fuente: INEN

\section{Colores.}

Su oficio tener cuidado, mostrar la existencia de un riesgo y facilitar su caracterización fácil y rápidamente franjas de las señales donde se aplica el color son tres:

- Franja de seguridad.

- Franja de contraste.

- Franja de símbolo. (JOSE, 2012, pág. 3)

No toda la luz emitida por una fuente luminosa, por ejemplo, una bombilla, es percibida por el ojo humano, ni toda la energía consumida por esa fuente se transforma en luz. Para controlar esta serie de parámetros, es necesario esta- blecer nuevas magnitudes con las que se puedan realizar mediciones correctas. 
- Flujo luminoso $(\phi)$. "Potencia (W) emitida en forma de luz a la que el ojo humano es sensible".

La magnitud en la que se mide el flujo luminoso $(\phi)$ es el lumen (lum), a la relación entre el Watt y el lumen se la llama equivalente luminoso de la energía y equivale a:

- Intensidad luminosa (I). "Es el flujo luminoso emitido por unidad de ángulo sólido en una dirección concreta". El flujo luminoso nos dice la cantidad de luz que emite una fuente de luz. Dependiendo del tipo de fuente, la luz se emitirá en todas las direcciones o en una dirección determinada, por ejemplo, una bombilla emitirá la luz en todas las direcciones del espacio y un proyector lo hará solo en una dirección.

Está claro que es necesario saber cómo se distribuye el flujo luminoso en cada dirección mediante la intensidad luminosa (I), cuya unidad es la candela (cd).

- Iluminancia (E). "Es el flujo luminoso recibido por una superficie”. (JOSE, 2012)

\section{Señalización horizontal.}

La señal horizontal, concierne a la aplicación de marcas viales, accedidas por líneas, flechas, símbolos y letras que se pintan sobre el pavimento, bordillos o sardineles y estructuras de las vías de tráfico o contiguas a ellas, así como los objetos que se instalan sobre la superficie de rodadura, con el fin de regular, regularizar el tránsito o indicar la apariencia de obstáculos.

\section{Consideraciones generales.}

La demarcación desempeña funciones definidas e importantes en un adecuado esquema de regulación del tránsito. En algunos casos, son usadas para completar las disposiciones o avisos de otros terminales, tales como las indicaciones verticales y semáforos; en otros, participan ilustraciones que no pueden ser mostradas mediante el uso de todo otro dispositivo, siendo un modo muy efectivo de hacerlas entendibles.

Para que la señalización horizontal cumpla la función para la cual se usa, se requiere que se tenga una igualdad respecto a las extensiones, diseño, insignias, caracteres, colores, repetición de uso, circunstancias en que se utiliza y tipo de material pasado.

Las diferencias viales o demarcaciones incumben ser reflectivas excepto marcha peatonal ejemplar cebra, o estar ajustadamente iluminadas.

Las figuras de demarcación con boceto en frío que se apliquen sobre concreto asfáltico corresponderán quedar pintadas como mínimo treinta (30) días después de labrada en la carpeta de rodadura. Cuando por contextos específicos se solicite realizar el territorio antes de dicho término, ésta tocará realizarse empleando un espesor húmedo igual a la mitad del descrito para la pintura definitiva y se deberá colocar aquella adentro de los ocho (8) días siguientes. 


\section{Materiales.}

Las estampillas viales se deben crear mediante el uso de pinturas en frío o en caliente. Sin embargo, puede preparar otro tipo de material, siempre que efectúe con las especificaciones de color y claridad; siendo preciso que no muestren contextos resbalosos, fundamentalmente en los pasos peatonales y en las cercanías a éstos.

Los cuadros alargados, lograrán estilarse dispositivos particulares (tachas, estoperoles o boceto termoplástica con pequeños abultamientos-vibraline), que resalten menos de 2,5 $\mathrm{cm}$ del área de la tierra y de color blanco o amarillo.

Hacia delimitar cabos o islas, conseguirán manejar nuevos dispositivos (tachones, boyas resistente o plásticas, bordillos, etc.), que descuellen del área del suelo a una altura máxima de $10 \mathrm{~cm}$.

Los requerimientos que debe verificar el boceto en frío para marcación de pavimentos son los vistos en la norma técnica colombiana NTC-1360-1.

las fallas reflectivas corresponderán efectuar con lo especificado en la norma técnica colombiana NTC-4745.

Los requerimientos para el bosquejo y estudio de materiales como pinturas, termoplásticos, dúctiles en frío y hiladillos preformadas, empleados en la demarcación de vías y vías, son los establecidos en la norma técnica colombiana NTC-4744.

\section{Colores y letras.}

Las figuras longitudinales y estampillas corresponden ser blancas o amarillas. En las líneas alargados el color blanco se empleará para crear apartamiento entre tránsito en el igual sentido y el amarillo entre tránsito de sentido inverso. Las flechas, emblemas y letras serán de color blanco, a excepción de las flechas de doble cabeza manipuladas para la demarcación de carriles de contraflujo. Cuando se solicite dar oposición a las figuras blancas o amarillas podrá utilizar líneas obscurecidas adyacentes a ellas y de amplio parejo a $1 / 2$ del ancho de la raya, excepto para estampillas viales en donde se realizarán líneas negras que resalten $5 \mathrm{~cm}$, la demarcación de suelos se traerán las letras del alfabeto contenido en el actual capítulo.

\section{Clases de señales de tránsito.}

Reglamentarias o prescriptivas

- Prohibición: simbolizan que determinada acción no puede realizarse.

- Restricción: son aquellas que indican los límites de la velocidad, peso y tamaño de los vehículos, uso de estacionamiento y carriles.

- Prioridad: cambian o refuerzan la prioridad respecto de a quién corresponde pasar primero en una esquina o tramo del camino. 


\section{Señales informativas.}

- Nomenclatura urbana (destinos y distancias, características de la vía).

- Información turística.

- Servicios.

\section{Señales preventivas.}

- Máximo peligro: indican que hay que conducirse con extrema precaución sobre determinados lugares porque el peligro que hay alrededor es muy grande.

- Físicas: simbolizan determinadas características de la ruta, por ejemplo: que se acerca una curva, un túnel o un puente. (mtop, s.f.)

\section{Clasificación.}

La señalización horizontal se clasifica así:

- Estampillas longitudinales.

- Estampillas transversales.

- Estampillas de bordillos y sardineles.

- Estampillas de objetos.

- Adentro de la vía.

- Contiguos a la vía.

\section{Marcas longitudinales.}

Raya continua sobre la calzada figura que ningún conductor con su carro debe cruzar ni circular sobre ella, ni cuando la estampilla separe los dos sentidos de tráfico, circular por la izquierda de ella.

Marca longitudinal formada por dos líneas incesantes tiene el mismo significado. Se exceptúan a importante las líneas continuas de borde de calzada.

\section{Líneas centrales.}

Se utilizarán estas líneas de color amarillo, para mostrar el eje de una calzada con tránsito en las dos vías y de color blanco para apartar carriles de tránsito, en el mismo sentido. En contextos especiales esta raya puede no estar en el centro geométrico de la pista, como es el caso de mutaciones en el ancho del pavimento, cuando hay un carril adherido para partida lenta, en la entrada a túneles o puentes angostos, etc.

Las líneas centradas corresponden utilizar en los siguientes casos:

- En vías rurales de dos molestos, con ancho de pavimento de 5,50 m o más. 
- En vías suplentes o de jerarquía superior, adentro del perímetro urbano de las poblaciones.

- En todas las vías o carreteras de cuatro o aumento de carriles, ciclorrutas, autopistas, carreteras principales y secundarias, y todas las vías en donde un artículo de ingeniería de tránsito así lo aconseje.

Las figuras centrales estarán accedidas por una línea segmentada de $12 \mathrm{~cm}$ de ancho, como mínimo, con una relación de distancias entre segmento y espacio de tres (3) a cinco (5).

Tendrán las siguientes dimensiones:

\section{En vías rurales.}

- Longitud del segmento pintado 4,50 m.

- Longitud del espacio sin pintar 7,50 m.

\section{En vías urbanas.}

- Longitud del segmento pintado 3,00 m.

- Longitud del espacio sin pintar 5,00 m.

Líneas centrales y de borde de pavimento.

Línea de borde de pavimento.

Línea central.

\section{Gráficos de borde de pavimento.}

Esta raya separa la acera del carril de tráfico, indicando el borde exterior del pavimento las vías, urbanas y rurales que no reciten con sardineles y en las vías arterias o de jerarquía gigante, se debe demarcar la orilla de suelo para frenar el tráfico de vehículos por la acera y fundamentalmente en el acercamiento a encuentros, cruces, puentes angostos, contornos urbanos, etc.

La línea de orilla de suelo de color amarillo a la derecha de la calzada, en vías con separador, indica la finalización de circulación en ese sentido.

También podrán demarcarse líneas de borde de pavimento de color azul, en las aproximaciones a hospitales, clínicas y centros de atención médica. Estas líneas se teñirán en las vías que lleven a tales sitios, desde una distancia de $500 \mathrm{~m}$ o más. Los casos en que se diferencie mantener la raya de borde de pavimento de color blanco, se instalarán tachas reflectivas bidireccionales de color azul, apartadas entre sí $3 \mathrm{~m}$. 


\section{Líneas del carril.}

Las líneas aprovecharán para concretar los carriles que llevan el tránsito en igual dirección. Asimismo, cumplen lo puesto de aumentar la eficacia del uso de una vía en sitios en donde se muestran congestionamientos.

Hay que indicar que la permuta del carril se puede hacer sin enfrentar un riesgo, se usará una línea blanca fraccionada de $12 \mathrm{~cm}$ de ancho, como mínimo, con correspondencia de longitudes entre fragmento y espacio de tres (3) a cinco (5), conforme a las consecutivas dimensiones:

\section{En vías rurales.}

- Longitud del segmento pintado 4,50 m

- Longitud del espacio sin pintar 7,50 m

\section{En vías urbanas.}

- Longitud del segmento pintado 3,00 m.

- Longitud del espacio sin pintar 5,00 m.

El canje de carril puede conducir un riesgo, si no se verifica con precaución, se usará una línea blanca perpetua de $12 \mathrm{~cm}$ de ancho, como mínimo.

\section{Dibujos de separación de rampas de entrada o de salida.}

Realizan la separación entre el carril de tráfico de una vía de alta ligereza y la rampa de entrada o de salida, donde existen huellas de prontitud o desaceleración para los vehículos. Las líneas serán de color blanco, discontinuos con tramos de un metro $(1,0 \mathrm{~m})$, apartadas un metro $(1,0 \mathrm{~m}) \mathrm{y}$ con un ancho de $0,20 \mathrm{~m}$.

Figura 2. Señalización Horizontal

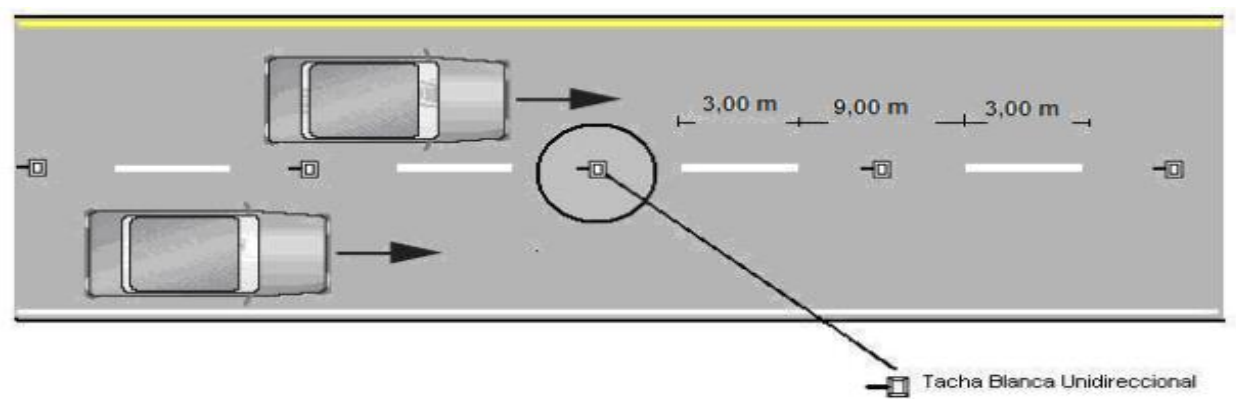

Fuente: INEN, Líneas de carriles segmentadas, Reglamento Técnico Ecuatoriano. 
Por el nivel de riesgo que implica esta maniobra las zonas de no rebasar deben estar debidamente señalizadas según el reglamento de la ley orgánica de transporte terrestre tránsito y seguridad vial, es decir en todos aquellos sitios en los que exista "una distancia de visibilidad de rebasamiento menor a la distancia de rebasamiento mínimo, siendo esta última la distancia necesaria para que el vehículo abandones su carril, pase al vehículo que lo procede y retorne a su carril de forma segura, sin afectar la velocidad del vehículo rebasado, ni la del otro que se desplaza del sentido contrario del carril utilizado para rebasar” Reglamento técnico ecuatoriano de Señalización. (INEN, 2011)

Es conveniente para lugares entrañables donde se requiera señalizar escapatorias de urgencia, escaleras, pasamanos, caminos de seguridad, líneas de evacuación con el propósito de a seguridad, durante la pérdida de luz natural o artificial. El efecto luminiscente tiene una durabilidad de hasta 2 horas. (Pinturas Unidas, 2018)

Figura 3. Efecto de la aplicación de la pintura Fotoluminiscente

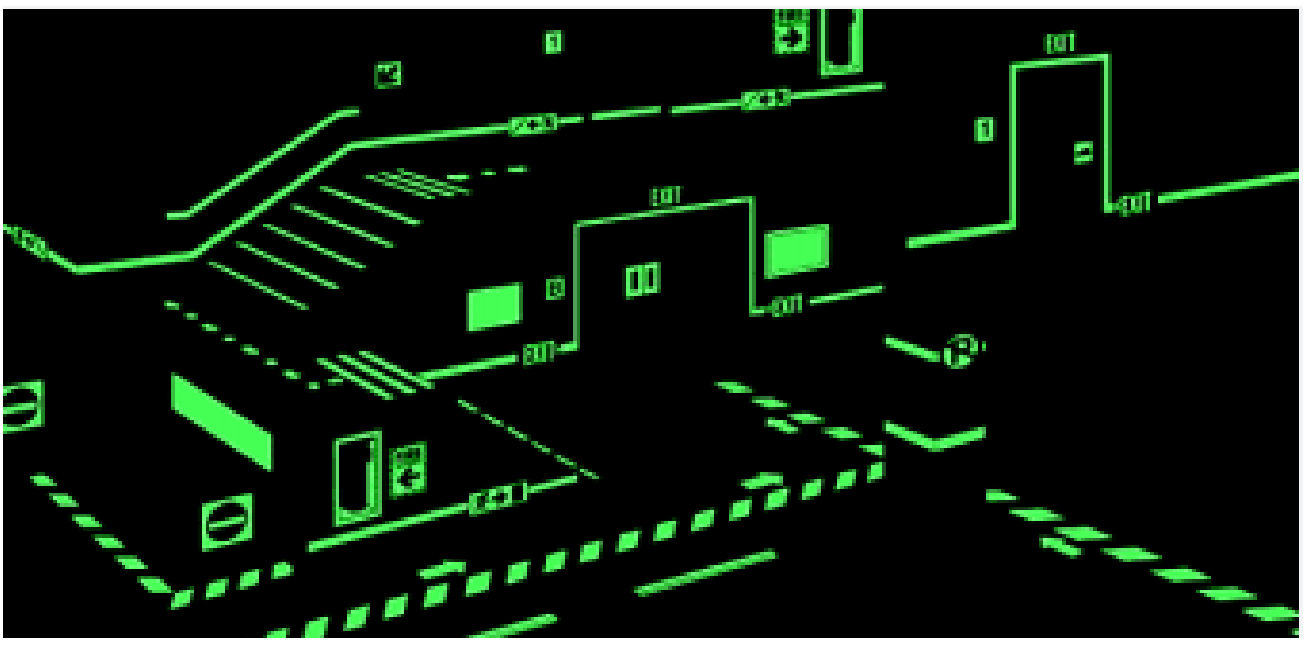

Fuente: Pinturas unidas.

\section{Metodología de la investigación.}

\section{Tipo de investigación.}

El trabajo investigativo será de tipo experimental se manipula las variables, mediante observación y análisis de las vías, donde el investigador presencia el problema y mediante las técnicas e instrumentos necesarios comprobará la idea a defender planteada.

\section{Tipo de estudio.}

Se aplicará un estudio Transversal, debido a que no existe continuidad en el eje del tiempo, es decir que se mide a la vez la prevalencia de la exposición y del efecto en una muestra poblacional en un solo momento temporal.

\section{Métodos de investigación.}


- Método Inductivo: Este método parte de lo particular a lo general, ya que inicia con la observación de los problemas individuales de las vías para poder llegar a conclusiones generales sobre la realidad de esta.

- Método Analítico: A través de este método se analizará cada resultado obtenido para detectar las posibles causas que ocasionan falencias.

\section{Técnicas de la investigación.}

Las técnicas empleadas para la recolección de información son:

- Encuestas: Para la presente investigación se pretende recolectar información directa de los accionistas, la misma que dará a conocer las opiniones, actitudes y sugerencias.

- Observación directa: Para el desarrollo de este proyecto el equipo de investigación obtiene información directa de la aplicación de la pintura directamente en las vías señaladas.

\section{Instrumentos.}

- Cuestionario: La aplicación de una encuesta con preguntas cerradas y abiertas dirigidas a los usuarios de las vías determinando sus expectativas y percepción.

- Ficha de observación: Se utiliza un check list para tiempos, calidad y duración de las pinturas, objeto de estudio junto con la calidad de las vías.

\section{Alcance del proyecto.}

Este análisis tiene la finalidad de generar una correcta señalización horizontal prototipo dentro de la movilidad interna de la ESPOCH. Con líneas específicas de dos vías principales, que dará lugar a otros proyectos.

\section{Desarrollo de la investigación.}

Nuestra investigación se basará en los siguientes parámetros:

\section{Métodos, técnicas e instrumento.}

Métodos.

Científico.

Caracterizado por tener un conjunto de pasos para realizar un experimento y de ahí obtener una respuesta o conclusión de este.

\section{Inductivo.}

Método que obtiene conclusiones generales a partir de premisas particulares; lo que se debe analizar los aspectos generales tanto variables dependientes e independientes. 


\section{Deductivo.}

Requiere de principios generales, conocimientos bastos sobre el tema para llegar a cualquier conclusión de orden particular.

Lo que en la investigación mediante un razonamiento lógico deducirá por qué mejorar el servicio de calidad y el funcionamiento del sistema operacional del terminal.

\section{Analítico.}

Este método implica el análisis es decir la separación de un todo en sus partes o elementos constitutivos.

Lo que permitirá hacer el análisis del sistema operacional del terminal Inter cantonal para lograr una mejor funcionalidad al mismo.

\section{Sintético.}

Un proceso analítico - sintético el cual se parte de la tesis de casos, biografías o fenómenos individuales para llegar al hallazgo de un iniciación o ley que rige. En el proyecto de investigación es la orden y agrupación de datos para desarrollar las conclusiones respectivas.

\section{Técnicas.}

\section{Observación directa.}

Que para una apreciación y/o análisis de la confusa, lo recomendable es estar en relación directo con campo de estudio, para realizar una base de datos de todos los aspectos que influyen en la respectiva problemática, a efecto de que nos sirva de elementos de disposición a formular el análisis, conclusiones, recomendaciones y propuestas.

\section{Encuesta.}

Se empleará esta técnica, la misma que será dirigida a los conductores y usuarios.

\section{Instrumento.}

\section{Cuestionario.}

Se realiza como una herramienta para la realización de las encuestas donde constarán preguntas abiertas y cerradas proporcionando información necesaria.

\section{Ficha de observación.}

Son instrumentos de la investigación de campo. Se usan cuando el investigador debe registrar datos que aportan otras fuentes como son personas, grupos sociales o lugares donde se presenta la problemática. (Baldereola, 2009) 


\section{Resultados.}

Análisis e interpretación de resultados.

Ficha de observación de las vías de la Espoch.

Tabla 2. Situación actual.

\begin{tabular}{|c|c|c|c|c|}
\hline \multirow{2}{*}{ Descripción } & \multirow{2}{*}{ Existe } & \multirow{2}{*}{ No existe } & \multirow{2}{*}{$\mathbf{N}^{\circ}$} & Dimensiones \\
\hline & & & & $\mathbf{L} \quad \mathbf{A}$ \\
\hline $\begin{array}{l}\text { Señalización } \\
\text { horizontal }\end{array}$ & $\mathrm{Si}$ & & & \\
\hline $\begin{array}{l}\text { Señalización } \\
\text { vertical }\end{array}$ & $\mathrm{Si}$ & & & \\
\hline $\begin{array}{l}\text { Visualización } \\
\text { de señalética }\end{array}$ & $\mathrm{Si}$ & & & \\
\hline $\begin{array}{l}\text { Se encuentran } \\
\text { correctamente } \\
\text { ubicados }\end{array}$ & & & & \\
\hline $\begin{array}{l}\text { Servicios de } \\
\text { seguridad }\end{array}$ & No & & & \\
\hline
\end{tabular}

Fuente: Elaboración propia.

Tabla 3. Ficha de observación de las vías de la Es poch con el uso de la Pintura

\begin{tabular}{lllll}
\hline Descripción & Si & No & $\mathbf{N}^{\circ}$ & \multicolumn{2}{c}{ Dimensiones } \\
\hline $\begin{array}{l}\text { Señalización } \\
\text { horizontal } \\
\text { clara }\end{array}$ & $\mathrm{X}$ & & \\
$\begin{array}{l}\text { Visualización } \\
\text { a distancia }\end{array}$ & $\mathrm{X}$ & & \\
$\begin{array}{l}\text { Visualización } \\
\text { de color } \\
\text { apropiado }\end{array}$ & $\mathrm{X}$ & & \\
$\begin{array}{l}\text { Se encuentran } \\
\text { correctamente } \\
\text { ubicados }\end{array}$ & $\mathrm{X}$ & & \\
$\begin{array}{l}\text { Visible para } \\
\text { el conductor }\end{array}$ & $\mathrm{X}$ & & \\
\hline
\end{tabular}

Fuente: Elaboración propia. 


\section{Características de la pintura.}

- Anteriormente da aplicar mezclar perfectamente la pintura con una paleta limpia, desde el fondo del recipiente hacia fuera, gestionando homogenizar todo el material incluso que esté libre de lugares y tenga un color uniforme.

- Este producto al exterior puede trastornar totalmente la funcionalidad del producto.

- El beneficio de la pintura podrá modificar debido al tipo de área a pintar, arruga y porosidad de la superficie, procedimiento de aplicación usado, situaciones de trabajo, grosor de película, desperdicios de pintura, etc.

- El máximo efecto luminiscente, deberá siempre utilizarse un fondo color blanco.

El uso de otro color no está recomendado ya que puede perjudicar el desempeño de la pintura Acrílica Fotoluminiscente.

- El efecto fotoluminiscente depende del tipo de sustrato, tipo de luz, tiempo de exposición y ángulo de incidencia. La aplicación incorrecta del fondo y/o de la pintura provocará una mala funcionalidad.

- El efecto fotoluminiscente puede ser afectado por suciedad o contaminación.

\section{Conclusiones:}

- Propiedades físicas y datos de aplicación. Color: Verde luminiscente Acabado: Mate Exposición: Solo para interior Sólidos por masa: 55\% Sólidos por volumen: 45\% Peso específico: $1.18 \mathrm{~g} / \mathrm{cm}^{3}$ Número de componentes: Uno Viscosidad a $25^{\circ} \mathrm{C}$ : 60 - 65 Seg / Copa Ford No 4 Proporción de le mezcla: Se aplica sin diluir Tiempo de vida útil en el envase: 1 año Espesor seco recomendado: 50 micras Rendimiento teórico: $8.0 \mathrm{~m}^{2}$ / litro a 50 micras de espesor Entintado: No se entinta Método de aplicación: Pistolas: sin aire (Airless) convencional Brocha y Rodillo Presión de boquilla: 175 bar Limpieza de equipos: Reductor Cód 403 Tiempo de secado al tacto a $25^{\circ} \mathrm{C} 30$ min Para repintar: 2 horas Tiempo de luminiscencia: 2 horas. (Pinturas Unidas, 2018)

- Al analizar las propiedades físicas de la pintura primeramente iniciaríamos por el costo en relación con la pintura tradicional su costo es alto. El pavimento tiene que ser totalmente liso como se lo demuestra a continuación.

- Preparación de la superficie. Es adecuado para lugares interiores donde se requiera señalizar salidas de emergencia, escaleras, pasamanos, caminos de seguridad, líneas de evacuación con la finalidad de proporcionar seguridad, durante la pérdida de luz natural o artificial. El efecto luminiscente tiene una durabilidad de hasta 2 horas. 
- Toda superficie antes de recubrirla debe de cumplir con las siguientes condiciones. - Limpia: Elimine polvo, grasa, pintura suelta, óxido, moho, tierra, y cualquier material que impida que la pintura se adhiera a la superficie. Remueva con cepillo de alambre toda la pintura suelta o dañada. - Seca: No deberá existir humedad en la superficie. - Opaca: La superficie no deberá ser brillante ni estar pulida. - Superficies mampostería, concreto, cemento y materiales similares: Estas superficies deberán tener por lo menos 28 días de haber sido curadas.

- Lave la superficie con una solución al $10 \%$ de ácido muriático. Deje actuar por 20 minutos. Enjuague la superficie con abundante agua hasta eliminar el exceso de ácido. Deje secar perfectamente la superficie. Repare grietas y huecos con un resonador para muros.

- Nuestras carreteras tienen grietas es decir su efecto no conviene, en otros países si utilizan para la aplicación de señalización horizontal pero la realidad es otra con respecto a nuestros países.

\section{Referencias bibliográficas:}

Baldereola, d. (2009). metodología de la investigación.

Campillos López, J. (2012). Mantenimiento del sistema de alumbrado y señalización (MF0627_2). Málaga, Spain: IC Editorial. Recuperado de https://elibro.net/es/ereader/espoch/42703?page=14

Castro, F. C. (2017). manual del transportista. España: Ediciones Diaz de Santos.

Cerda, I. (2017). logística urbana. Spain: marge Books.

https://www.pinturasunidas.com/uploads/productos/industrial/21.PINTURA\%20FOTO

$\begin{array}{llll}\text { LUMINISCENTE.pdf. } & \text { (s.f.). }\end{array}$ https://www.pinturasunidas.com/uploads/productos/industrial/21.PINTURA\%20 FOTOLUMINISCENTE.pdf:

https://www.pinturasunidas.com/uploads/productos/industrial/21.PINTURA\%20 FOTOLUMINISCENTE.pdf

INEN. (2011). Zonas de no rebasar.

INEN. (s.f.). ángulos de iluminación. INEN.

INEN. (s.f.). líneas de carriles segmentadas (Reglamento técnico Ecuatoriano). INEN. Recuperado el jueves de junio de 21018

JOSE, C. L. (jueves de abril de 2012). Mantenimiento Del Sistema De Alumbrado. España: IC Editorial. Recuperado el jueves de abril de 2018, de https:///senalizacion.html: html.rincondelvago.com/senalizacion.html 
Mtop. (s.f.). Señales de tránsito Ecuador. Obtenido de .forosecuador.ec/forum/aficiones/autos-y-motos/9146-señales-de-tránsito-deecuador-y-su-significado-informativas-preventivas-reglamentarias

Rodriguez, D., \& Valldeoriola, J. (2009). Metodología de Investigación. Barcelona: Universitat Oberta de Catalunya. 
PARA CITAR EL ARTÍCULO INDEXADO.

Llamuca Llamuca, J. L., Salas Salazar, M. del R., \& Guerrero Godoy, A. P. (2020). Prueba piloto de señalización en la Escuela Superior Politécnica del Chimborazo pintura

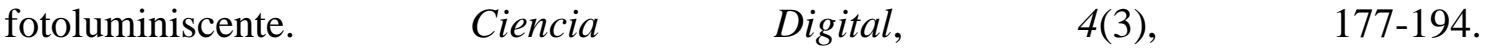
https://doi.org/10.33262/cienciadigital.v4i3.1319

\section{Ciencia \\ LDigital}

El artículo que se publica es de exclusiva responsabilidad de los autores y no necesariamente reflejan el pensamiento de la Revista Ciencia Digital.

El artículo queda en propiedad de la revista y, por tanto, su publicación parcial y/o total en otro medio tiene que ser autorizado por el director de la Revista Ciencia Digital.
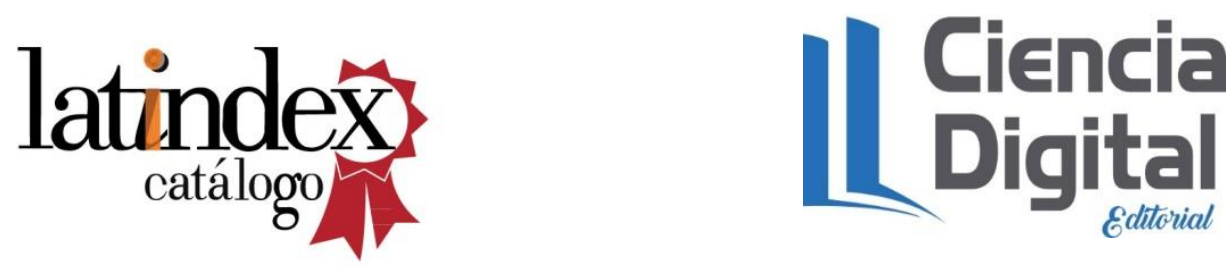